\title{
Preface to the special issue "The International Union of Geodesy and Geophysics: from different spheres to a common globe"
}

\author{
Jo Ann Joselyn ${ }^{1, *}$ and Alik Ismail-Zadeh ${ }^{2,3}$ \\ ${ }^{1}$ Space Environment Center, National Oceanic and Atmospheric Administration, Boulder, Colorado, USA \\ ${ }^{2}$ Karlsruhe Institute of Technology, Institute of Applied Geophysics, Karlsruhe, Germany \\ ${ }^{3}$ Russian Academy of Sciences, Institute of Earthquake Prediction Theory and Mathematical Geophysics, \\ Moscow, Russia \\ * retired
}

Correspondence: Alik Ismail-Zadeh (alik.ismail-zadeh@kit.edu)

Published: 16 April 2019

Many environmental challenges facing society since the last century require international solutions. Examples include exploration of the Earth and its environment in space; exploration of our rivers, oceans, and atmosphere; climate change; securing clean water and energy; and understanding and mitigating disasters due to natural hazard events. Earth and space sciences lie at the heart of these challenges and occupy a central role in international agendas.

The International Union of Geodesy and Geophysics (IUGG) has been coordinating and promoting international efforts in Earth and space sciences since 1919. Under IUGG's umbrella, eight international scientific associations and several interdisciplinary bodies cover many disciplines of geo- and space sciences and promote research of the Earth from its core and its space environment up to the Sun. Thousands of scientists from many nations and specific scientific disciplines have developed ways of cooperation through IUGG's international associations and learned how to work together to promote geosciences. IUGG has been initiating, developing, and implementing international cooperative programs; setting scientific standards; developing research tools; educating and building capacity; and contributing to science for policy and science diplomacy.

The purpose of this special issue (SI) is not to relay a definitive history of the development of international cooperation in the geosciences, but to summarize IUGG's remarkable role during the 100-year span of its existence. The first part of the SI "The union: bringing together geophysical disciplines" begins with the present overview of the IUGG mission and structure, and the circumstances of its founding and early development are presented up to the beginning of World War II. The second article starts with recovery after World War II and addresses the years of extraordinary development of geophysical science through the International Geophysical Year (IGY; 1957-1958) and the concurrent evolution of IUGG structure and programs extending up to the General Assembly of 1999. The rapid advances that have occurred thus far into the 21st century are reviewed in the third article, and foresights on IUGG future development conclude this first part of the SI. Outstanding histories of each of the IUGG associations are then told.

The second part of the SI "Around the earth: glaciers, rivers, air, and oceans" presents articles about the histories of the International Association of Cryospheric Sciences (IACS: past, present, and future of the International Association of Cryospheric Sciences), the International Association of Hydrological Sciences (IAHS: a brief history of hydrology), the International Association of Meteorology and Atmospheric Sciences (IAMAS: a century of international cooperation in atmospheric sciences), and the International Association for the Physical Sciences of the Oceans (IAPSO: tales from the ocean frontier).

The third part of the SI "Within and on the earth: gravity, magnets, earthquakes, and volcanoes" presents articles about the histories of the International Association of Geodesy (The International Association of Geodesy: from an ideal sphere to an irregular body subjected to global change), the International Association of Geomagnetism and Aeronomy 
(IAGA: a major role in understanding our magnetic planet), the International Association of Seismology and Physics of the Earth's Interior (IASPEI: its origins and the promotion of global seismology), and the International Association of Volcanology and Chemistry of the Earth's Interior (IAVCEI: from small beginnings to a vibrant international association).

This volume, proposed in 2014 by the IUGG Bureau, is the combined effort of a number of authors and advisors, including past and current IUGG and association officers. We are grateful to Franz Kuglitsch and Katina Rogers Roopchansingh for their technical and editorial assistance, to Hans Volkert for proposing the title of this SI and editorial support, and to reviewers for their constructive comments and suggestions for revision of the initial manuscripts of the papers published in the SI. The IUGG archives, curated by the American Institute of Physics Center for the History of Physics (Gregory Good, director), located in College Park, MD, USA, were heavily consulted in preparation for this SI, and we acknowledge their helpful staff. We are very thankful to Kristian Schlegel, HGSS editor-in-chief, for his enthusiastic efforts and assistance in producing the SI.

Jo Ann Joselyn and Alik Ismail-Zadeh

SI Editors 
Appendix A: List of acronyms

\begin{tabular}{|c|c|}
\hline AfSC & African Seismological Commission \\
\hline AGU & American Geophysical Union \\
\hline AI & artificial intelligence \\
\hline AK1345 & seismic travel-time model, without special meaning \\
\hline AMS & Army Map Service \\
\hline AOGS & Asia Oceania Geosciences Society \\
\hline ASC & Asian Seismological Commission \\
\hline BAS & British Antarctic Survey \\
\hline $\mathrm{BCE}$ or $\mathrm{BC}$ & Before Common Era \\
\hline BGI & Bureau Gravimétrique International \\
\hline BHI & Bureau International de l'Heure \\
\hline BIPM & Bureau International des Poids et Mesures \\
\hline BV & Bulletin Volcanologique (Bulletin of Volcanology) \\
\hline CAST & China Association for Science and Technology \\
\hline CCEC & Commission on Climatic and Environmental Change (IUGG) \\
\hline CCTF & Consultative Committee for Time and Frequency \\
\hline $\mathrm{CFC}$ & chlorofluorocarbon \\
\hline CIESM & Commission Internationale pour l'Exploration Scientifique de la mer Méditerranée \\
\hline CIG & Comite Internationale de Geophysique \\
\hline CIG & Commission Internationale des Glaciers \\
\hline CliC & Climate and Cryosphere project (WCRP) \\
\hline CME & coronal mass ejection \\
\hline CMG & Commission on Mathematical Geophysics (IUGG) \\
\hline CMIP & Coupled Model Intercomparison Project \\
\hline CMSLT & Commission on Mean Sea Level and Tides \\
\hline CNAA & Committee on Nucleation and Atmospheric Aerosols (of ICCP) \\
\hline CNES & Centre National d'Etudes Spatiales, France \\
\hline $\mathrm{CO}_{2}$ & carbon dioxide \\
\hline CODATA & Committee on Data for Science and Technology \\
\hline COSPAR & Committee on Space Research \\
\hline COSTED & Committee on Science and Technology in Developing Countries \\
\hline $\mathrm{CoV}$ & Cities on Volcanoes \\
\hline COWAR & Committee on Water Research \\
\hline CRCM & Commission on Recent Crustal Movements \\
\hline CSAGI & Committee for the International Geophysical Year \\
\hline CSR & Committee on Space Research (IUGG) \\
\hline CSTG & Coordination of Space Techniques for Geodesy and Geodynamics \\
\hline CTBT & Comprehensive Nuclear-Test-Ban Treaty \\
\hline DACA-13 & Davos Atmosphere and Cryosphere Assembly, 2013 \\
\hline $\mathrm{DC}$ & District of Columbia \\
\hline DFG & German Research Foundation \\
\hline DMI & Danish Meteorological Institute \\
\hline DOES & Deep Ocean Exchange with the Shelf \\
\hline DORIS & Doppler Orbitography and Radiopositioning Integrated by Satellite \\
\hline EC & Executive Committee \\
\hline ECR & early career researcher \\
\hline ECS & early career scientist \\
\hline ECV & essential climate variable \\
\hline ED50 & European Datum 1950 \\
\hline EGIG & Expédition Glaciologique Internationale au Groenland 1957-1960 \\
\hline eGY & electronic Geophysical Year \\
\hline EMSEV & Inter-Association Working Group on Electromagnetic Studies of Earthquakes and V \\
\hline
\end{tabular}


ENHANS Extreme Natural Hazards and Societal Implications

ENSO El Niño-Southern Oscillation

EOS-80 International Equation of State of Seawater - 1980

EOST Ecole et Observatoire des Sciences de la Terre, Strasbourg

ESA European Space Agency

ESC European Seismological Commission

ESM Earth System Model

FAGS Federation of Astronomical and Geophysical Data Analysis Services

FAO The Food and Agriculture Organization of the United Nations

FDSN International Federation of Digital Seismograph Networks

FIG Fédération Internationale de Géomètres

FRG Federal Republic of Germany

G7

GA Group of Seven countries: Canada, France, Germany, Italy, Japan, UK, and USA

GAPHAZ Glacier and Permafrost Hazards in Mountains standing group (IACS \& IPA)

GARP Global Atmospheric Research Program

GCOS Global Climate Observing System

GDP International Geodynamics Project

GDR German Democratic Republic

GEBCO General Bathymetric Chart of the Oceans

GEO Group on Earth Observations

GEOSS Global Earth Observation System of Systems

GEWEX Global Energy and Water Exchanges project (WCRP)

GFZ

GGOS

GGRF

GGP

GIS

GLASS

German Research Centre for Geosciences

GLIMS

Global Geodetic Observing System

Global Geodetic Reference Frame

Global Geodynamics Project

GLOF

GLOSS

GNSS

Geographic Information System

GOOS

GEWEX Land Atmosphere System Study

GPS

GRC

GRS 67

Global Land Ice Measurements from Space initiative

glacier lake outburst flood

GRS 80

Global Sea Level Observing System

Global Navigation Satellite Systems

GRACE

Global Ocean Observing System

GTN-G

GVP

Global Positioning System

HMS

HMSH

HSJ

Commission on Geophysical Risk and Sustainability (IUGG)

IABO

IACS

Geodetic Reference System 1967

Geodetic Reference System 1980

IAG

IAGA

Gravity Recovery And Climate Experiment

IAGC

IAGP

Global Terrestrial Network for Glaciers (GCOS)

Global Volcanism Program (SI)

IAHR

His/Her Majesty's Ship

His Most Serene Highness

Hydrological Sciences Journal (IAHS)

IAHS

IAM

International Association for Biological Oceanography

International Association of Cryospheric Sciences (IUGG)

International Association of Geodesy (IUGG)

International Association of Geomagnetism and Aeronomy (IUGG)

IAMAP
IAMAS

International Association of Geochemistry and Cosmochemistry

International Antarctic Glaciological Project

International Association of Hydraulic Research

International Association of Hydrological Sciences (IUGG)

International Association for Meteorology (IUGG); previous name for IAMAS

International Association of Meteorology and Atmospheric Physics (IUGG); previous name for IAMAS

International Association of Meteorology and Atmospheric Sciences (IUGG) 
IAPO International Association of Physical Oceanography (IUGG); previous name for IAPSO

IAPSO International Association for the Physical Sciences of the Oceans (IUGG)

IAPWS International Association for the Properties of Water and Steam

IASC International Arctic Science Committee

IASH International Association of Scientific Hydrology (IUGG); previous name for IAHS

IASPEI International Association of Seismology and Physics of the Earth's Interior (IUGG)

IASPEI91 seismic travel-time model, without special meaning

IASY International Years of the Active Sun

IATME International Association of Terrestrial Magnetism and Electricity (IUGG); previous name for IAGA

IAU International Astronomical Union

IAV International Association of Volcanology (IUGG); previous name for IAVCEI

IAVCEI International Association of Volcanology and Chemistry of the Earth's Interior (IUGG)

ICACGP International Commission of Atmospheric Chemistry and Global Pollution (IAMAS)

ICAE International Commission on Atmospheric Electricity (IAMAS)

ICAO International Civil Aviation Organization

ICCE International Commission on Continental Erosion (IAHS)

ICCL International Commission on Climate (IAMAS)

ICCLAS International Commission on the Coupled Land-Atmosphere System

ICCP International Commission of Clouds and Precipitation (IAMAS)

ICCT Inter-Commission Committee on Theory (IAG)

ICDM International Commission on Dynamical Meteorology (IAMAS)

ICES International Council for the Exploration of the Sea

ICET International Centre for Earth Tides

ICGEM International Centre for Global Earth Models

ICGW International Commission on Groundwater (IAHS)

ICMA International Commission on the Middle Atmosphere (IAMAS)

ICPAE International Commission on Planetary Atmospheres and their Evolution (IAMAS)

ICPM International Commission on Polar Meteorology (IAMAS)

ICRCM International Centre on Recent Crustal Movements

ICRS International Commission on Remote Sensing (IAHS)

ICSH International Commission on Statistical Hydrology (IAHS)

ICSI International Commission on Snow and Ice (IAHS)

ICSIH International Commission on Snow and Ice Hydrology (IAHS)

ICSU International Council for Science; formerly, International Council of Scientific Unions

ICSW International Commission on Surface Water (IAHS)

ICT International Commission on Tracers

ICTP Abdus Salam International Centre for Theoretical Physics

ICWQ International Commission on Water Quality (IAHS)

ICWRS International Commission on Water Resources Systems (IAHS)

IDEMS International Digital Elevation Model Service

IDNDR International Decade for Natural Disaster Reduction

IDS International DORIS Service

IEEY International Equatorial Electrojet Year

IERS International Earth Rotation Service

IGAC International Global Atmospheric Chemistry Experiment

IGBP International Geosphere-Biosphere Programme

IGC International Geological Congress

IGEMS Integrated Global Earth Monitoring System

IGeS International Geoid Service

IGETS International Geodynamics and Earth Tide Service

IGFS International Gravity Field Service

IGRF International Geomagnetic Reference Field

IGS International GNSS Service (previously International GPS Geodynamics Service,

International Service for Geodynamics)

IGS International Glaciological Society 


\begin{tabular}{|c|c|}
\hline IGSN 71 & International Gravity Standardization Net 1971 \\
\hline IGY & International Geophysical Year \\
\hline IHA & International Hydrology Association \\
\hline IHD & International Hydrological Decade \\
\hline IHDP & International Human Dimensions Programme \\
\hline IHP & International Hydrological Programme \\
\hline IHY & International Heliophysical Year \\
\hline IIOE-2 & Second International Indian Ocean Expedition \\
\hline iLEAPS & Integrated Land Ecosystem-Atmosphere Processes Study \\
\hline ILP & International Lithosphere Program \\
\hline ILRS & International Laser Ranging Service \\
\hline ILS & International Latitude Service \\
\hline IMO & International Meteorological Organization; former name for the WMO \\
\hline IOC & Intergovernmental Oceanographic Commission \\
\hline $\mathrm{IOC}$ or $\mathrm{IO}_{3} \mathrm{C}$ & International Ozone Commission (IAMAS) \\
\hline IOS & UK Institute of Oceanographic Sciences \\
\hline ION & International Ocean Network \\
\hline IPA & International Permafrost Association \\
\hline IPCC & International Panel on Climate Change \\
\hline IPGH & Instituto Panamericano de Geografia Historia (also PAIGH) \\
\hline IPMS & International Polar Motion Service \\
\hline IPY & International Polar Year \\
\hline IPYDIS & IPY Data and Information Service \\
\hline IQSY & International Years of the Quiet Sun \\
\hline IRC & International Research Council \\
\hline IRC & International Radiation Commission (IAMAS) \\
\hline IRDR & Integrated Research on Disaster Risk \\
\hline ISA & International Seismological Association \\
\hline ISC & International Science Council \\
\hline ISC & International Seismological Centre (UK) \\
\hline ISG & International Service for the Geoid \\
\hline ISGI & International Service of Geomagnetic Indices \\
\hline ISO & International Organization for Standardization \\
\hline ISS & International Seismological Summary \\
\hline ISSC & International Social Science Council \\
\hline ITD & inter- and trans-disciplinary \\
\hline ITRF & International Terrestrial Reference Frame \\
\hline ITU & International Telecommunications Union \\
\hline IUBS & International Union of Biological Sciences \\
\hline IUCI & Inter-Union Commission on the Ionosphere \\
\hline IUCRM & Inter-Union Commission on Radio Meteorology \\
\hline IUCSTP & Inter-Union Commission on Solar-Terrestrial Physics \\
\hline IUGG & International Union of Geodesy and Geophysics \\
\hline IUGS & International Union of Geological Sciences \\
\hline IUPAC & International Union of Pure and Applied Chemistry \\
\hline IUPAP & International Union of Pure and Applied Physics \\
\hline IVS & International VLBI Service for Geodesy and Astrometry \\
\hline IY & International Year \\
\hline IYD & International Year of Deltas \\
\hline IYGU & International Year of Global Understanding \\
\hline IYPE & International Year of the Planet Earth \\
\hline JCS & The Joint Committee on the Properties of Seawater \\
\hline JPOTS & Joint Panel on Oceanographic Tables and Standards \\
\hline KIT & Karlsruhe Institute of Technology, Germany \\
\hline
\end{tabular}




\begin{tabular}{|c|c|}
\hline LACSC & Latin American and Caribbean Seismological Commission \\
\hline LOICZ & Land-Ocean Interactions in the Coastal Zone \\
\hline MAP & Middle Atmosphere Programme \\
\hline MEMS & micro-electro-mechanical systems \\
\hline MOCA-09 & Meteorology, Ocean and Cryosphere Assembly, held in Montreal in 2009 \\
\hline $\begin{array}{l}\text { MOCA-21 } \\
\text { m.s.l. }\end{array}$ & $\begin{array}{l}\text { Meteorology, Ocean and Cryosphere Assembly, to be held in Busan, Republic of Korea, in } 2021 \\
\text { mean sea level }\end{array}$ \\
\hline NASA & National Aeronautics and Space Administration (USA) \\
\hline NMSOP-2 & New Manual of the Seismological Observatory Practice (second edition) \\
\hline NNR NUVEL-1A & No Net Rotation Northwestern University Velocity model 1A \\
\hline NSIDC & National Snow and Ice Data Center (USA) \\
\hline OGO & Orbiting Geophysical Observatory \\
\hline OSIL & Ocean Scientific International Ltd. \\
\hline PAIGH & Pan-American Institute of Geography and History (also IPGH) \\
\hline $\mathrm{PC}$ & personal computer \\
\hline PIAHS & Proceedings of IAHS \\
\hline PIOSA & Pan-Indian Ocean Science Association \\
\hline POGO & Polar Orbiting Geophysical Observatory \\
\hline PRC & People's Republic of China \\
\hline PREM & Preliminary Reference Earth Model (IASPEI) \\
\hline PSA & Pacific Science Association \\
\hline PSFG & Permanent Service on the Fluctuations of Glaciers \\
\hline PSMSL & Permanent Service for Mean Sea Level \\
\hline PSS-78 & Practical Salinity Scale 1978 \\
\hline PUB & Prediction in Ungauged Basins program (IAHS) \\
\hline RETrig & Réseau Européen de Triangulation \\
\hline REUN & Réseau Européen Unifié de Nivellement \\
\hline ROC & Republic of China \\
\hline ROSTSEA & UNESCO Regional Office for Science and Technology for Southwest Asia \\
\hline SA & scientific assembly \\
\hline SCAR & Scientific Committee on Antarctic Research \\
\hline SCL & Scientific Committee on the Lithosphere \\
\hline SCOPE & Scientific Committee on Problems of the Environment \\
\hline SCOR & Scientific Committee on Ocean Research \\
\hline SCOSTEP & Scientific Committee on Solar-Terrestrial Physics \\
\hline SCOWAR & Scientific Committee on Water Research \\
\hline SDG & Sustainable Development Goal \\
\hline SDO & Solar Dynamics Observatory \\
\hline SEAN & Scientific Event Alert Network \\
\hline SEDI & Commission on Study of Earth's Deep Interior (IUGG) \\
\hline SFE & solar flare effect \\
\hline SHS & Section d'Hydrologie Scientifique (IUGG) \\
\hline SI & Smithsonian Institute for Natural Sciences \\
\hline SLR & satellite laser ranging \\
\hline SnowMIP & Snow Model Intercomparison Project \\
\hline $\mathrm{SOHO}$ & Solar and Heliospheric Observatory \\
\hline SOLAS & Surface Ocean-Lower Atmosphere Study \\
\hline SP6 & seismic travel-time model, without special meaning \\
\hline SPARC & Stratosphere-troposphere Processes And their Role in Climate \\
\hline$S_{\mathrm{R}}$ & Reference Salinity \\
\hline SSC & storm sudden commencement \\
\hline SST & Sea surface temperature \\
\hline SV & Section of Volcanology (IUGG) \\
\hline TEOS-10 & Thermodynamic Equation of Seawater - 2010 \\
\hline
\end{tabular}




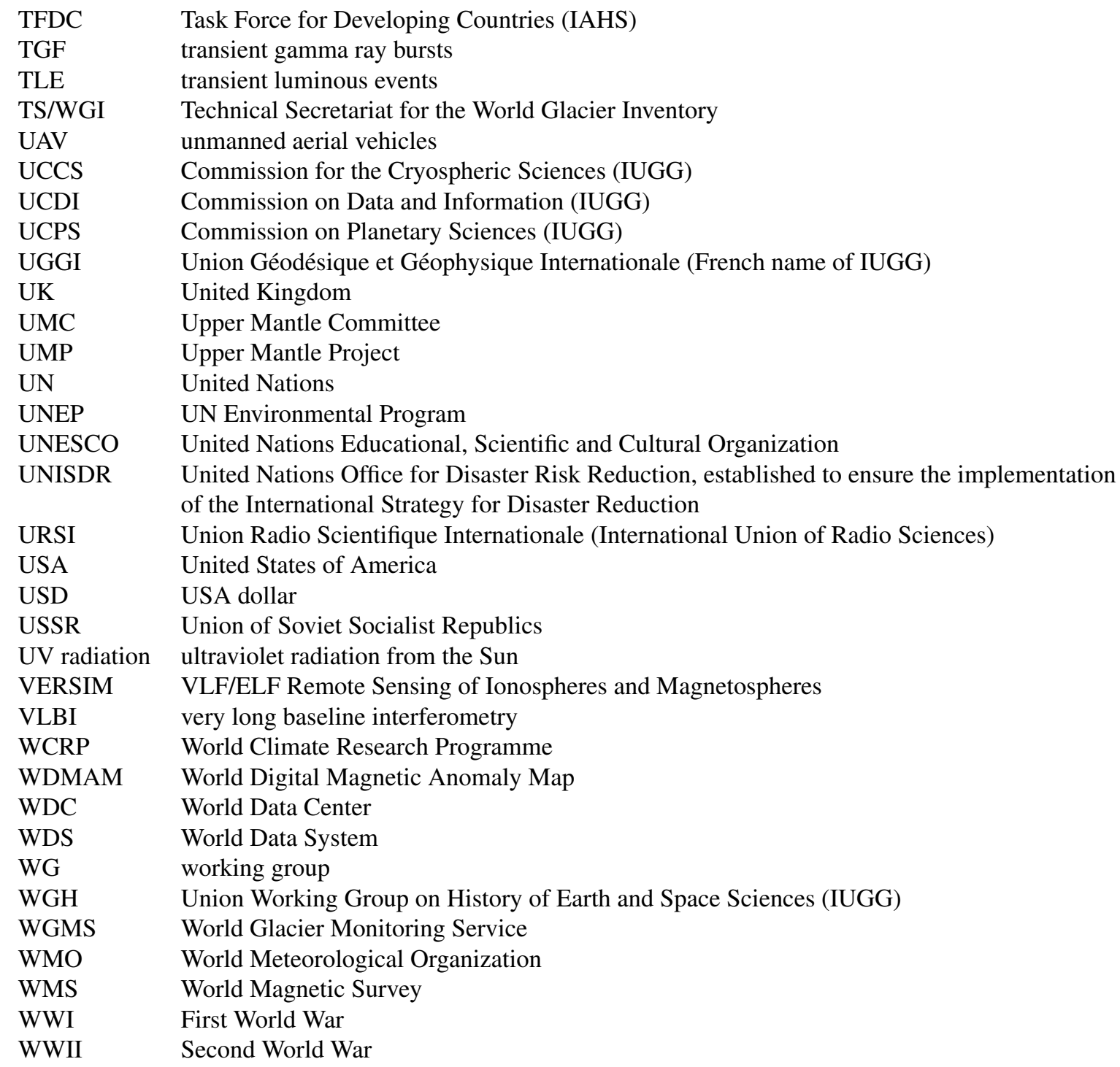

